\title{
Phlegmonous Gastritis: A Report of Three Cases with Clinical and Imaging Features
}

\author{
Mitsuaki Ishioka, Noboru Watanabe, Masayuki Sawaguchi, Sho Fukuda, Hisashi Shiga, \\ Tamotsu Matsuhashi, Mario Jin and Katsunori Iijima
}

\begin{abstract}
:
Phlegmonous gastritis is a rare but often fatal acute pyogenic infection of the stomach. We herein report three cases of phlegmonous gastritis with different causes: the long-term placement of a nasogastric feeding tube, bacteremia associated with cellulitis in a diabetic patient, and an adverse reaction to paclitaxel/carboplatin chemotherapy for cancer of unknown primary cause, which were classified as primary, secondary, and idiopathic types, respectively. Coping with the increasing morbidity rate associated with the diverse background of such patients requires a thorough understanding of the clinical features and image findings associated with this entity.
\end{abstract}

Key words: phlegmonous gastritis, nasogastric feeding tube, cellulitis, paclitaxel/carboplatin chemotherapy

(Intern Med 57: 2185-2188, 2018)

(DOI: 10.2169/internalmedicine.0707-17)

\section{Introduction}

Phlegmonous gastritis is an uncommon but often fatal acute pyogenic infection of the stomach. Although the mortality rate has been reduced with the improvement of antibiotic therapy, a majority of patients are still diagnosed after gastrectomy or autopsy $(1,2)$. According to most previous reports, mucosal injury due to gastric cancer or peptic ulcer is regarded as the main etiology of phlegmonous gastritis. However, a diverse background of these patients has been noted in recent reports, suggesting that that the range of clinical features and image findings of this entity should be noted. We herein report three cases of phlegmonous gastritis with different causes: the long-term placement of a nasogastric feeding tube, bacteremia associated with cellulitis in a diabetic patient, and an adverse reaction to paclitaxel/carboplatin chemotherapy for cancer of unknown primary cause (CUP).

\section{Case Reports}

\section{Case 1}

Early in November 2017, an 84-year-old woman with an advanced stage of dementia was transported to our emergency room because of the obvious flow of fresh blood from her nasogastric feeding tube. Her temperature was $37.4^{\circ} \mathrm{C}$, and the laboratory findings showed an elevated serum C-reactive protein (CRP) level of $13.6 \mathrm{mg} / \mathrm{dL}$. Emergency esophagogastroduodenoscopy (EGD) revealed extensive gastric ulceration with necrotic tissue and an edematous mucosa (Fig. 1A and B). Subsequent abdominal computed tomography (CT) showed a thickened gastric wall with an intramural low-density area thought to be an abscess. Proteus mirabilis and $\alpha$-streptococcus were isolated from a culture of gastric juice and biopsies. Following a diagnosis of phlegmonous gastritis, sulbactam/ampicillin (3 g/day) was started intravenously. One week later, EGD was performed again and showed a significant improvement (Fig. 1C and D). Four weeks later, we confirmed the complete healing by EGD (Fig. 1E and F), and the patient was discharged from our hospital. 


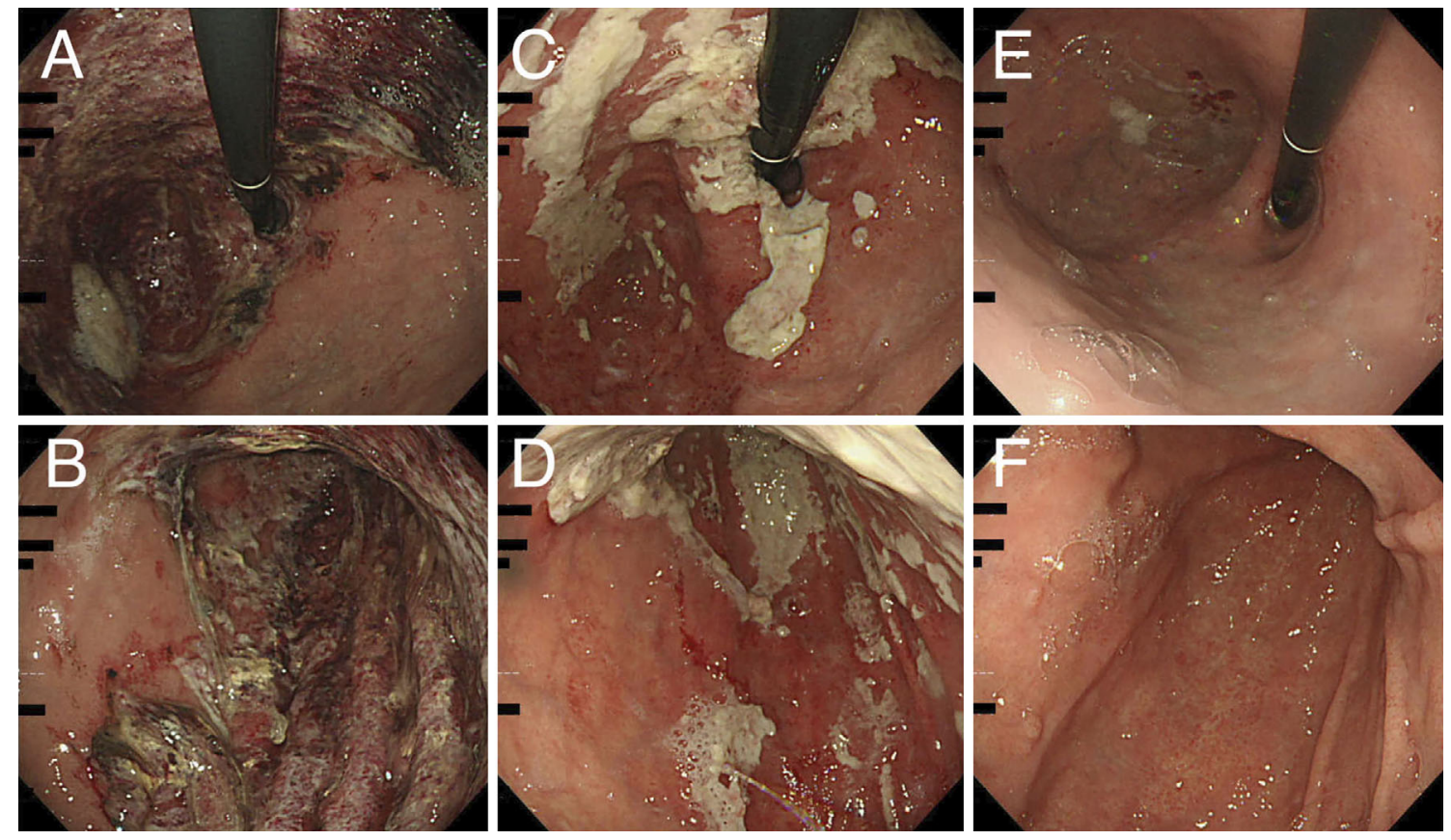

Figure 1. The EGD findings in case 1. (A, B) On the day of admission. (C, D) The healing stage at one week after admission. (E, F) The remission stage at four weeks after admission. EGD: esophagogastroduodenoscopy

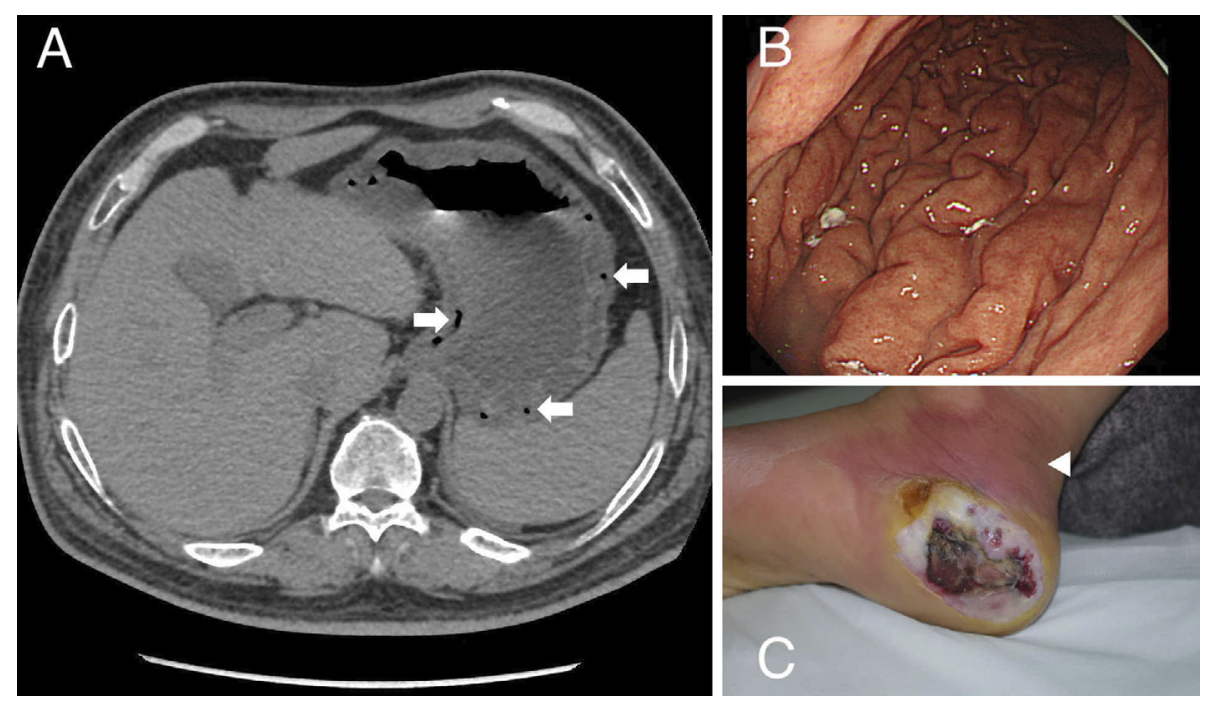

Figure 2. Case 2. (A) The CT image at onset. The white arrows indicate abscesses in the thickened gastric wall. (B) The EGD image showing thickened gastric folds. (C) Cellulitis of the left foot. The arrowhead shows redness near the skin ulcer. EGD: esophagogastroduodenoscopy

\section{Case 2}

A 44-year-old man presented with a 4-day history of nausea and epigastric pain early in April 2017. He had a history of uncontrolled type 2 diabetes for 10 years and had undergone treatment for diabetic foot gangrene. As an increase in the white blood cell (WBC) count $\left(12.1 \times 10^{9}\right.$ cells $\left./ \mathrm{L}\right)$ and CRP level (8.93 mg/dL) was observed, chest-abdomenpelvis CT was conducted, and a thickened gastric wall with an intramural low-density area were detected (Fig. 2A). Subsequent EGD revealed markedly thickened gastric folds and redness (Fig. 2B) that appeared to be normal after the 9-day intravenous administration of ceftriaxone ( $2 \mathrm{~g} /$ day). Notably, in this case, Staphylococci were isolated from a culture of both gastric biopsies and diabetic gangrene. The following month, he developed diabetic cellulitis of the leg and bacteremia caused by Staphylococci again (Fig. 2C), but abdominal pain did not recur.

\section{Case 3}

The patient was a 64-year-old man introduced to our hospital at the beginning of March 2017 due to CUP. His fam- 


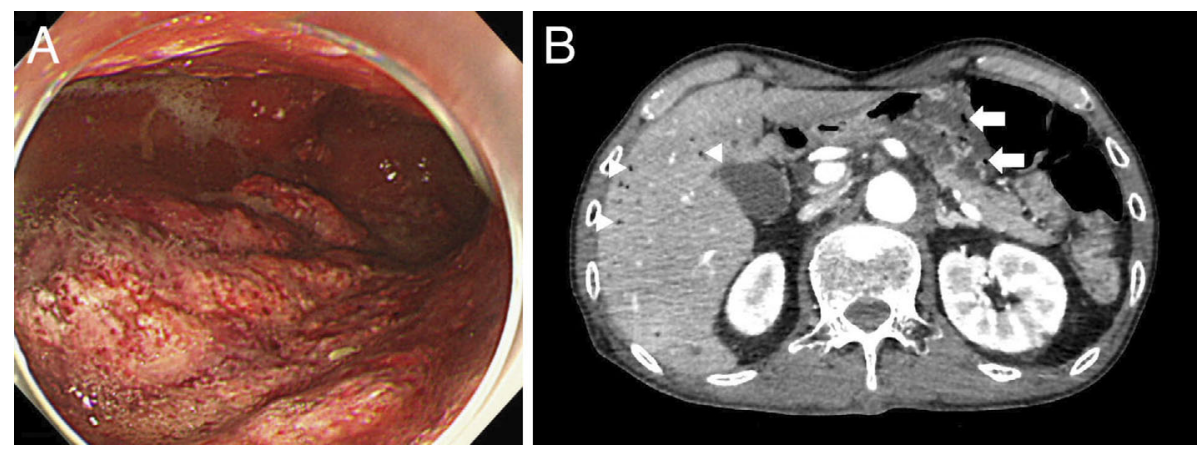

Figure 3. Case 3. (A) The EGD image at onset. (B) The CT image at onset. The white arrows indicate abscesses in the thickened gastric wall. The arrowhead indicates portal venous pneumatosis. EGD: esophagogastroduodenoscopy

ily doctor initially found a brain tumor as the cause of his six-month history of gradually deteriorating numbness in the right hand. Whole-body CT in our hospital revealed multiple lesions in the lung, liver, ribs, and lymph nodes. The upper/ lower gastrointestinal tract was intact at this time. On consideration of the histological findings, including immunohistochemistry from a right axillary lymph node biopsy, poorly differentiated lung adenocarcinoma was highly suspected. First-line treatment with paclitaxel $\left(200 \mathrm{mg} / \mathrm{m}^{2}\right)$ and carboplatin (area under the curve 5) every 3 weeks was started. Four days after the first administration of chemotherapy, nausea and hematemesis occurred. Emergency EGD showed a dark-purple, friable, and thickened mucosa (Fig. 3A) that improved after the 7-day intravenous administration of cefepime ( $2 \mathrm{~g} /$ day). Air in the thickened gastric wall with concomitant portal venous pneumatosis was seen on $\mathrm{CT}$ at onset (Fig. 3B). Candida colonization was noted in gastric biopsy specimens. Chemotherapy was changed to the oral administration of tegafur-gimeracil-oteracil potassium $(100 \mathrm{mg} /$ day); however, he died due to tumor progression 4 months after admission.

\section{Discussion}

Phlegmonous gastritis is classified into primary, secondary, and idiopathic types $(3,4)$. Primary phlegmonous gastritis is the most common type and it is normally caused by mucosal injury due to gastric cancer or peptic ulcer. Conversely, secondary phlegmonous gastritis occurs in association with a systemic infection, such as endocarditis (5). A diagnosis of "idiopathic" disease is made without mucosal injury or any apparent infection focus. Most "idiopathic" cases occur in compromised hosts. Our three cases had primary, secondary, and idiopathic disease (one each). Case 1 had primary phlegmonous gastritis caused by mucosal irritation due to a nasogastric feeding tube, and Case 2 had secondary phlegmonous gastritis caused by bacteremia associated with cellulitis in a diabetic patient. It is difficult to describe case 3 as strictly "idiopathic", as the observed phlegmonous gastritis was an adverse reaction to paclitaxel/carboplatin chemotherapy for CUP. However, given that there was no mucosal injury in precritical EGD nor any other infection focus, we deemed the diagnosis to thus be "idiopathic". To our knowledge, there are only a few single reports of phlegmonous gastritis caused by the placement of a feeding tube $(6,7)$, and this is first report of phlegmonous gastritis subsequent to cellulitis or paclitaxel/carboplatin chemotherapy, but these are all common backgrounds.

Regardless of its etiology, the clinical features and image findings of phlegmonous gastritis shared by most cases are as follows: the sudden onset of a fever, epigastric pain, nausea and hematemesis with severe inflammation; a thickened gastric wall with an intramural low-density area on CT; and fold thickening or extensive ulceration on EGD. Given that it can often be misdiagnosed as peptic ulcer perforation, Borrmann type 4 gastric cancer or gastric malignant lymphoma from CT/EGD alone, it is important to judge all of these elements together to make an accurate diagnosis of phlegmonous gastritis $(8,9)$.

Streptococcus spp. is isolated as the causative agent in approximately $70 \%$ of all cases followed by Staphylococcus spp., Escherichia coli, and the other indigenous bacteria in the digestive tract $(2,10)$. In the present cases, indigenous bacteria in the digestive tract, Proteus mirabilis, $\alpha$ streptococcus, Staphylococci, and Candida, were isolated from either gastric juice or biopsy specimens. An elevated gastric $\mathrm{pH}$ is known to be a risk factor of phlegmonous gastritis $(1,11)$. Interestingly, a recent study indicated that proton pump inhibitors (PPIs) alter the gut microbiota and increase the growth of pathogens of phlegmonous gastritis, such as Streptococcus spp., Staphylococcus spp., and Escherichia coli $(12,13)$. In our cases 1 and 3 , PPIs had been prescribed for over a year. PPI usage is also reported to be a risk factor of spontaneous bacterial peritonitis in cirrhotic patients (14). In these previous reports, gastric acid was thought to play a crucial role in preventing the invasion of pathogens, and PPIs induce a dysfunction of the mucosal barrier (11-14). For this reason, phlegmonous gastritis can also occur as an adverse event associated with direct mucosal injury, such as that induced by endoscopic submucosal dissection and endoscopic ultrasound-guided fine-needle aspiration $(15,16)$. 
In conclusion, phlegmonous gastritis is a rare condition which occurs due to common causes. Its morbidity may subsequently increase due to the following backgrounds: an aging society, increasing prevalence of diabetes mellitus, overuse of PPIs, more aggressive endoscopic techniques, and the development of new chemotherapies. Having a good knowledge of its clinical features and imaging findings is indispensable to make an early diagnosis and improve the treatment outcomes.

The authors state that they have no Conflict of Interest (COI).

\section{References}

1. Miller AI, Smith B, Rogers AI. Phlegmonous gastritis. Gastroenterology 68: 231-238, 1975.

2. Kim GY, Ward J, Henessey B, et al. Phlegmonous gastritis: case report and review. Gastrointest Endosc 61: 168-174, 2005.

3. Konjetzny GE. Die entzündungen des magens. In: Handbuch der Speziellen Pathologischen Anatomie und Histologie, Vol. 4. 2nd ed. Henke F, Lubarsch O, Eds. Springer, Berlin, 1928: 768-1116 (in German).

4. Soga K, Kassai K, Itani K, Yagi N, Naito Y, Itoh Y. Gastric outlet obstruction induced by a gastric wall abscess after cholecystitis. Intern Med 53: 2675-2678, 2014.

5. LaForce FM. Diffuse phlegmonous gastritis. A rare complication of pneumococcal endocarditis. Arch Intern Med 120: 230-233, 1967.

6. Zenooz NA, Robbin MR, Perez V. Gastric pneumatosis following nasogastric tube placement: a case report with literature review. Emerg Radiol 13: 205-207, 2007.

7. Bashour CA, Popovich MJ, Irefin SA, et al. Emphysematous gas- tritis. Surgery 123: 716-718, 1998.

8. Jung K, Park MI, Kim SE, Park SJ. Borrmann type 4 advanced gastric cancer: focus on the development of scirrhous gastric cancer. Clin Endosc 49: 336-345, 2016.

9. Kussin SZ, Henry C, Navarro C, Stenson W, Clain DJ. Gas within the wall of the stomach report of a case and review of the literature. Dig Dis Sci 27: 949-954, 1982.

10. Rada-Palomino A, Muñoz-Duyos A, Pérez-Romero N, et al. Phlegmonous gastritis: a rare entity as a differential diagnostic of an acute abdomen. Description of a case and a bibliographic review. Rev Esp Enferm Dig 106: 418-424, 2014.

11. Turner MA, Beachley MC, Stanley D. Phlegmonous gastritis. AJR Am J Roentgenol 133: 527-528, 1979.

12. Imhann F, Bonder MJ, Vich Vila A, et al. Proton pump inhibitors affect the gut microbiome. Gut 65: 740-748, 2016.

13. Jackson MA, Goodrich JK, Maxan ME, et al. Proton pump inhibitors alter the composition of the gut microbiota. Gut 65: 749-756, 2016.

14. Ratelle M, Perreault S, Villeneuve JP, Tremblay L. Association between proton pump inhibitor use and spontaneous bacterial peritonitis in cirrhotic patients with ascites. Can J Gastroenterol Hepatol 28: 330-334, 2014

15. Ajibe $H$, Osawa H, Yoshizawa $M$, et al. Phlegmonous gastritis after endoscopic submucosal dissection for early gastric cancer. Therap Adv Gastroenterol 1: 91-95, 2008.

16. Itonaga $\mathrm{M}$, Ueda $\mathrm{K}$, Ichinose $\mathrm{M}$. Phlegmonous gastritis caused by endoscopic ultrasound-guided fine-needle aspiration (EUS-FNA). Dig Endosc 24: 488, 2012.

The Internal Medicine is an Open Access article distributed under the Creative Commons Attribution-NonCommercial-NoDerivatives 4.0 International License. To view the details of this license, please visit (https://creativecommons.org/licenses/ by-nc-nd/4.0/).

(C) 2018 The Japanese Society of Internal Medicine Intern Med 57: 2185-2188, 2018 